\title{
Profile of Fatal Suicide in Iran: A Report From the Iranian Forensic Medicine Between 2016 and 2018
}

\author{
Seyed Amirhosein Mahdavi ${ }^{1}$, Shahab Rezaeian ${ }^{2,3}$, Mehran Rostami ${ }^{4}$ \\ ${ }^{1}$ Legal Medicine Research Center, Legal Medicine Organization, Tehran, Iran \\ ${ }^{2}$ Infectious Diseases Research Center, Kermanshah University of Medical Sciences, Kermanshah, Iran \\ ${ }^{3}$ Clinical Research Development Center, Imam Reza Hospital, Kermanshah University of Medical Sciences, Kermanshah, Iran \\ ${ }^{4}$ Deputy of Health, Kermanshah University of Medical Sciences, Kermanshah, Iran
}

Received: 23 Jun. 2020; Accepted: 23 Oct. 2020

\begin{abstract}
The suicide rate among the Iranian population has increased over the past few decades. Descriptive information about this public health challenge could inform health policymakers to prioritize prevention strategies. We described the last updated data from the Iranian forensic medicine between 2016 and 2018. We obtained the data on the fatal suicide cases from the national suicide registry of the Iranian Forensic Medicine Organization (FMO), validated by death certificates and harmonized for epidemiologic studies. This study included 9,021 nationally registered suicide deaths from March 21, 2016, to March 20, 2018. A descriptive statistical approach was used to present the findings. Of 9,021 registered suicide deaths with age range between 10 to 94 years [median $=31], 44.6 \%[n=4,015]$ was in the category of $\leq 29,48.0 \%[n=4,328]$ between 30 to 59 , and $7.4 \%(n=666)$ in the category of $\geq 60$ years old. The percentage of fatal suicide was obviously higher in men $(71.2 \%, \mathrm{n}=6,424)$. Hanging was the most common method of suicide, both among men $(57.7 \%, \mathrm{n}=3706)$ and women $(37.6 \%, \mathrm{n}=976)$. The frequency of fatal suicide was significantly higher in married than single cases (52.6\% vs. $42.9 \%)$. Overall, at the national level, the most common suicide method was hanging (51.9\%), followed by self-poisoning (25.8\%) and self-immolation $(6.8 \%)$. We found evidence of age- and gender differences in suicide mortality across the country. Men, people in younger age groups, and married couples could be considered as the target population for preventive interventions in Iran.
\end{abstract}

(C) 2020 Tehran University of Medical Sciences. All rights reserved.

Acta Med Iran 2020;58(10):508-512.

Keywords: Iran; Mortality; Self-harm; Suicide method

\section{Introduction}

Suicide is one of the most important public health concerns all around the world, with about 800,000 death every year (1). Over $79 \%$ of global suicides occurred in developing countries, and it was the second leading cause of death among 15-29 years old in 2016 (1). World Health Organization (WHO) has been estimated that $2.4 \%$ of the global burden of diseases will be due to suicide in 2020 (2).

Suicide is a multifactorial phenomenon, and research revealed many risk factors for suicide, including demographic, social, cultural, and economic factors (2). Moreover, marital problems and family conflicts are the two well-known risk factors for suicide in Iranian families (3). In Iran, an increasing trend has shown in fatal suicide over the past few decades (4). Based on the global burden of diseases 2015 study (GBD 2015), suicide was ranked as the top ten leading causes of years of life lost (YLL) in Iran (5). However, the suicide mortality rate was close to five per 100,000 population in 2015 (6). This suicide mortality rate is lower than most countries in the world, but it is higher than the Eastern Mediterranean Region (EMR) average (7). Suicide methods vary across the regions and countries, but in the EMR, the three most common methods were hanging, self-poisoning, and selfimmolation (8). Suicide methods were associated with several factors such as gender, age, living area, education level, marital status, occupation, and availability of methods in Iran $(9,10)$.

An updated population-based comprehensive statistics from an authorized organization such as forensic medicine, which is directly involved with this health issue in Iran, is crucial for developing preventive measures at 
the national level. Subsequently, the purpose of this study was to report the current situation of fatal suicide in the Iranian population between 2016 and 2018.

\section{Materials and Methods}

\section{Data collection and registry}

The present cross-sectional study was a descriptive report of the fatal suicide data registered in the Iranian Forensic Medicine Organization (FMO) at the national level from March 21, 2016, to March 20, 2018. The Iranian FMO maintains a suicide registry, including all deaths due to suicide across the country. According to Iranian law and as a legal process to issue the death certificate, it is necessary to report all deaths due to external causes to FMO for forensic evaluation $(11,12)$. Moreover, all of the referred cases are subject to forensic autopsy; if the forensic test results reveal death by suicide, the case will be recorded in the national suicide registry (12). Therefore, it is acceptable to consider the fatal suicide registered cases by the FMO as the most valid registered data source for suicide in Iran (11-13).

We extracted relevant characteristics of fatal suicide cases from the Iranian FMO registry. During the past decade, based on a meta-analysis that recently conducted, the overall mean age of suicide in Iran was 28.9-year-old. The following information had extracted from the registry: Nationality, gender (male or female), age group (included $\leq 29$ years, 30-59 years, and $\geq 60$ years old), marital status (included single, married, widowed, divorced, separated, or unspecified), and the time of event including season of the year. The suicide methods were categorized as follows: hanging, self-poisoning (included intentional drug poisoning, pesticide poisoning, insecticide poisoning, and etc.), self-immolation, firearms, and other methods (included suicide by wristcutting, jumping from a height, suffocation, drowning, gassing, and other uncommon means). We found missing data for 10 cases and subsequently removed these cases from the current analysis. In addition, all other nationalities were excluded from the study.

\section{Statistical analysis}

Chi-square test was performed to compare the frequencies across categorical variables. We also used $t$ test to compare the mean age by genders. All statistical analysis has been done using Stata SE version 14 statistical package.

\section{Ethical and legal considerations}

We used anonymized data in order to conduct statistical analysis. The research protocol of the present study was reviewed and approved by the Ethics Committee of Kermanshah University of Medical Sciences in Iran (ethic number: IR.KUMS.REC.1398.330).

\section{Results}

From March 2016 to March 2018, a total of 9021 fatal suicides (6424 men and 2597 women) were registered in the Iranian FMO organization. Table 1 shows the demographical characteristics of fatal suicide cases overall and by gender. Of 9,021 fatal suicide cases, $71.2 \%$ were men, and $28.8 \%$ were women, with an age range from 10 to 94 years (median=31 yr). Overall, at the national level, the most common suicide method was hanging $(51.9 \%)$, followed by self-poisoning $(25.8 \%)$. The frequency of fatal suicide was significantly higher in married than single cases ( $52.6 \%$ vs. $42.9 \%)$.

Table 1. Demographic characteristics of fatal suicide cases reported from the Iranian forensic medicine between 2016 and 2018

\begin{tabular}{|c|c|c|c|c|c|}
\hline \multicolumn{2}{|c|}{ Characteristics } & $\begin{array}{c}\text { Total } \\
\text { No. }(\%) \\
\end{array}$ & $\begin{array}{l}\text { Women } \\
\text { No. }(\%)\end{array}$ & $\begin{array}{c}\text { Men } \\
\text { No. }(\%)\end{array}$ & $\boldsymbol{P}$ \\
\hline \multicolumn{2}{|c|}{$\leq 29$} & 4,015 (44.6) & $1,405(54.1)$ & $2,610(40.7)$ & \multirow{3}{*}{$<0.001$} \\
\hline group & & $4,328(48.0)$ & $1,042(40.2)$ & $3,286(51.2)$ & \\
\hline \multicolumn{2}{|c|}{$\geq 60$} & $666(7.4)$ & $148(5.70)$ & $518(8.1)$ & \\
\hline \multirow{4}{*}{$\begin{array}{l}\text { Suicide } \\
\text { methods }\end{array}$} & Hanging & $4,682(51.9)$ & 976 (37.6) & $3,706(57.7)$ & \multirow{4}{*}{$<0.001$} \\
\hline & Self-poisoning & $2,329(25.8)$ & 892 (34.6) & $1,437(22.4)$ & \\
\hline & Self-immolation & $614(6.8)$ & $364(14.0)$ & $250(3.9)$ & \\
\hline & Others* & $818(9.1)$ & 295 (11.3) & $523(8.1)$ & \\
\hline \multirow{3}{*}{ Marital status } & Single & $3,872(42.9)$ & $925(35.6)$ & $2,947(45.9)$ & \multirow{3}{*}{$<0.001$} \\
\hline & Married & $4,742(52.6)$ & $1,532(59.0)$ & $3,210(50.0)$ & \\
\hline & Others $\uparrow$ & $407(4.5)$ & $140(5.4)$ & $267(4.1)$ & \\
\hline \multirow{4}{*}{ Season } & Spring & $2,426(26.9)$ & $703(27.1)$ & $1,723(26.9)$ & \multirow{4}{*}{0.988} \\
\hline & Summer & $2,471(27.5)$ & $706(27.2)$ & $1,765(27.5)$ & \\
\hline & Fall & $2,026(22.5)$ & $583(22.5)$ & $1,443(22.5)$ & \\
\hline & Winter & $2,079(23.1)$ & $602(23.2)$ & $1,477(23.1)$ & \\
\hline
\end{tabular}

*Fall, wrist cutting, drowning, suffocation, starvation, and unusual methods of suicide

$\dagger$ Divorced, widowed, unknown 
The mean age of men was meaningfully higher than women (mean [SD] 35.8 [15.1] vs. 31.5 [14.4], $P<0.001$ ). We found that the prevalence of fatal suicide is significantly higher in women below 29 years $(54.1 \%)$ in comparison to men in the same age group (40.7\%), but in contrast, the prevalence was higher in middle age and above 60 years in men (Table 1). Moreover, we found a similar pattern of suicide methods between both genders, with hanging, self-poisoning, and self-immolation as the most prevalent method in both genders (Table 1). We did not find any observable pattern of fatal suicide by seasons (Table 1 and 2).

Figure 1 shows the absolute number of suicide cases by gender, age categories, and suicide methods. The stratified analysis showed the highest number of hanging in men across all age groups with an observable difference than other methods. While the number of cases who selected hanging and self-poisoning as the suicide methods was roughly similar in all age groups of women.

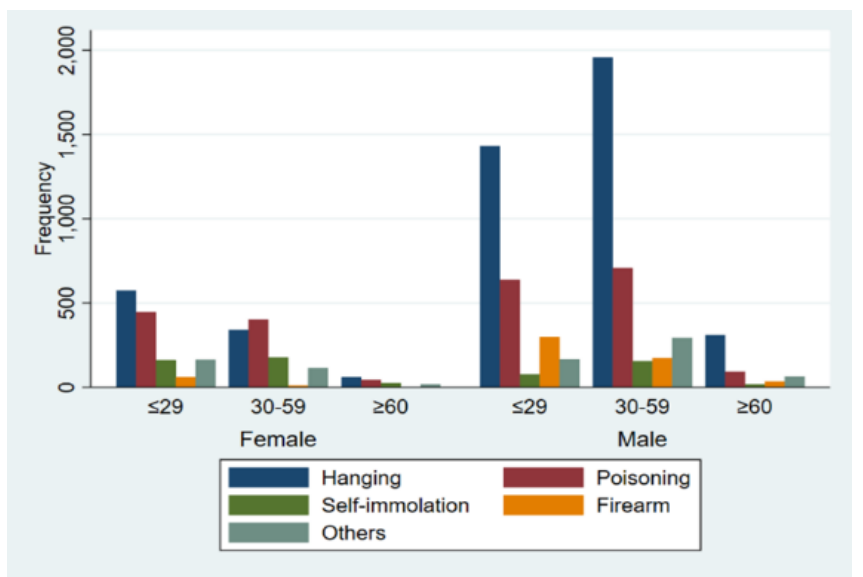

Figure 1. The absolute number of fatal suicide cases reported from the Iranian forensic medicine between 2016 and 2018, by age groups and gender

Table 2. Frequency of suicide methods and season reported from the Iranian forensic medicine between 2016 and 2018, by age groups

\begin{tabular}{|c|c|c|c|c|c|}
\hline & & $\begin{array}{c}\leq 29 \\
\text { No. }(\%)\end{array}$ & $\begin{array}{c}\text { 30-59 } \\
\text { No. }(\%)\end{array}$ & $\begin{array}{c}\geq 60 \\
\text { No. }(\%)\end{array}$ & $\boldsymbol{P}$ \\
\hline \multirow{5}{*}{$\begin{array}{l}\text { Suicide } \\
\text { methods }\end{array}$} & Hanging & 2,005 (49.9) & $2,297(53.1)$ & $370(55.6)$ & \multirow{5}{*}{$<0.001$} \\
\hline & $\begin{array}{l}\text { Self- } \\
\text { poisoning }\end{array}$ & $1,083(27.0)$ & 1,109 (25.6) & $136(20.4)$ & \\
\hline & $\begin{array}{l}\text { Self- } \\
\text { immolation }\end{array}$ & $239(6.0)$ & $332(7.7)$ & $43(6.5)$ & \\
\hline & Firearm & $360(9.0)$ & $183(4.2)$ & $35(5.3)$ & \\
\hline & Others & $328(8.1)$ & $407(9.4)$ & $82(12.3)$ & \\
\hline \multirow{4}{*}{ Season } & Spring & $1,043(26.0)$ & $1,191(27.6)$ & $188(28.3)$ & \multirow{4}{*}{0.20} \\
\hline & Summer & $1,082(27.0)$ & $1,198(27.8)$ & 191 (28.7) & \\
\hline & Fall & $904(22.5)$ & $956(22.1)$ & $162(24.4)$ & \\
\hline & Winter & $981(24.5)$ & $971(22.5)$ & $124(18.6)$ & \\
\hline
\end{tabular}

\section{Discussion}

This study provides comprehensive descriptive statistics of 9021 fatal suicide cases across Iran based on data registered at national FMO between 2016 and 2018. According to our findings, age- and gender differences were found in the suicide mortality. Over $71 \%$ of Iranian suicide cases were men. In addition, younger women (aged $\leq 29$ years) and adult men (aged 30-59 years) are reported as vulnerable subgroups in the Iranian population.

\section{Age and gender}

As already noted, our findings also indicating substantial age- and gender-inequality in suicide mortality across the country; a similar pattern has been 
reported both in the Iranian population $(6,12,14)$ and international studies $(15,16)$. Moreover, previous literature revealed that the socio-economic challenges $(9$, 14), family conflicts and marital problems (3), and risktaking behaviors including substance use disorders (17) could be the most important risk factors for suicide in the young adults' age categories.

\section{Marital status}

In our study, we found a high proportion of fatal suicide among married cases (around 53\%). There are conflicting findings on the role of marital status in fatal suicide. In traditional Iranian culture, as the dominant culture across the country, dealing with the experience of divorce-related-stigma is a big social and mental health challenge (10); thus, some couples may prefer to continue their marital relationship even when there are crucial marital related problems, and this might be one of the probable justification for our findings (10). However, evidence from other countries suggested that being nonmarried (never married, divorced/separated, or widowed) could increase the risk of suicide mortality $(9,18-20)$. Therefore, it seems that the role of marital status in fatal suicide could be highly dependent on the culture of communities and could be better to interpret in line with the socio-cultural specification of each population.

\section{Suicide methods}

In our study, the most common suicide method was hanging, followed by self-poisoning in both sexes and in all age groups. During 2006-2010, hanging was the most common method, followed by self-immolation and pesticide-poisoning in Iran (12). A recent study to assess suicide mortality in four provinces of Iran showed that hanging in men and self-immolation in women were the most frequent methods of suicide (21). Another study conducted on 1901 suicide cases to determine factors associated with the choice of suicide methods in the west of Iran showing the most common suicide methods of hanging, self-immolation, and intentional drug-poisoning in both sexes (10). The overview of suicide methods of WHO revealed that regional differences in the methods of suicide exist rather than between-country differences (22). In the EMR countries, people mostly hang themselves, $56 \%$ in Saudi Arabia (23), $48 \%$ in Turkey (24), $41 \%$ in Iraq (25), and over 50\% in Iran (12). In addition, Asian women rather completed suicide by hanging and by pesticide poisoning (26). This comparison may be suggested that horrific suicide methods such as self-immolation were replaced by other less violent methods.

\section{Seasonality}

There was no significant seasonal variation in the present study, which is not in line with the previous epidemiological studies in Iran. A national study showed a significant difference had been reported fatal suicide occurred in warm and cold seasons (27). Another regional study showed a significant seasonal variation with a peak in summer (28). The result of no significant seasonal variation appears as an external factor that is stochastic during the whole period. It is beyond the scope of this report to discuss the external factors that influence this seasonality.

This study has limitations. Considering conflicts with socio-cultural norms in the conservative context of Iran, it is likely that under-reporting and misclassification are probable problems for suicide data. Moreover, we did not report annual rates of suicide due to the lag between suicide occurrence and its registration in the national suicide record database. For example, the 2016 suicide database has some sharing cases that occurred in the previous years, like 2015 and 2014; or some of the suicide cases passed away in late 2016 would be registered in the database in 2017, and therefore unpredictable bias the annual rates of suicide is inevitable.

In conclusion, there was found evidence of age- and gender differences in suicide mortality across the country. Men, people in younger age groups, and married couples could be considered as the target population for preventive interventions in Iran. Health policymakers must seriously take into consideration for revision of suicide prevention programs. It can be understood that an informed decision about which strategy is best for preventing suicidal behaviors is very complex and multidimensional; educating at-risk individuals about coping skills (including problem-solving, support seeking, distress tolerance, and emotional regulation) as being one of the effective strategies at preventing suicidal behaviors.

\section{Acknowledgments}

The authors would like to thank Dr. Milad Nazarzadeh (University of Oxford, United Kingdom) for his useful comments. This study was financial supported by the Research Council of Kermanshah University of Medical Sciences (Grant Number: 980431 approved by Vice-Chancellor for Research Affairs of KUMS). The authors wish to thank the Iranian Legal Medicine Research Center for providing the data.

\section{References}


1. World Health Organization. Suicide. Geneva: Switzerland WHO press. (Accessed 2019 https://www.who.int/newsroom/fact-sheets/detail/suicide.)

2. Wasserman D, Wasserman C. Oxford textbook of suicidology and suicide prevention. USA: Oxford University Press, 2009.

3. Nazarzadeh M, Bidel Z, Ayubi E, Asadollahi K, Carson KV, Sayehmiri K. Determination of the social related factors of suicide in Iran: a systematic review and metaanalysis. BMC Public Health 2013;13:4.

4. Naghavi M, Shahraz S, Sepanlou SG, Dicker D, Naghavi P, Pourmalek F, et al. Health transition in Iran toward chronic diseases based on results of Global Burden of Disease 2010. Arch Iran Med 2014;17:321-35.

5. Moradi-Lakeh M, Sepanlou SG, Karimi SM, Khalili N, Djalalinia S, Karimkhani C, et al. Trend of sociodemographic index and mortality estimates in Iran and its neighbors, 1990-2015; findings of the global burden of diseases 2015 study. Arch Iran Med 2017;20:419-28.

6. Izadi N, Mirtorabi SD, Najafi F, Nazparvar B, NazariKangavari H, Hashemi-Nazari SS. Trend of years of life lost due to suicide in Iran (2006-2015). Int J Public Health 2018;63:993-1000.

7. Rezaeian M. Suicide among young middle eastern muslim females. Crisis 2010;31:36-42.

8. Morovatdar N, Moradi-Lakeh M, Malakouti SK, Nojomi M. Most common methods of suicide in Eastern Mediterranean Region of WHO: a systematic review and meta-analysis. Arch Suicide Res 2013;17:335-44.

9. Poorolajal J, Rostami M, Mahjub H, Esmailnasab N. Completed suicide and associated risk factors: a six-year population based survey. Arch Iran Med 2015;18:39-43.

10. Rostami M, Jalilian A, Rezaei-Zangeneh R, Salari A. Factors associated with the choice of suicide method in Kermanshah Province, Iran. Ann Saudi Med 2016;36:7-16.

11. Rostami M, Nazparvar B, Rezaeian S. Differences among official statistics of mortality rates in Iran. J Occup Health Epidemiol 2018;7:192-3.

12. Shojaei A, Moradi S, Alaeddini F, Khodadoost M, Abdizadeh A, Khademi A. Evaluating the temporal trend of completed suicides referred to the Iranian Forensic Medicine Organization during 2006-2010. J Forensic Legal Med 2016;39:104-8.

13. Karamouzian M, Rostami M. Suicide statistics in Iran: let's get specific. Am J Men's Health 2019;13:e1557988318807079.

14. Kiadaliri AA, Saadat S, Shahnavazi H, Haghparast-Bidgoli $\mathrm{H}$. Overall, gender and social inequalities in suicide mortality in Iran, 2006-2010: a time trend province-level study. BMJ open 2014;4:e005227.
15. Martini M, da Fonseca RC, de Sousa MH, de Azambuja Farias C, Cardoso TA, Kunz M, et al. Age and sex trends for suicide in Brazil between 2000 and 2016. Soc Psychiatry Psychiatr Epidemiol 2019;54:857-60.

16. Lee S, Dwyer J, Paul E, Clarke D, Treleaven S, Roseby R. Differences by age and sex in adolescent suicide. Aust N Z J Public Health 2019;43:248-53.

17. Poorolajal J, Haghtalab T, Farhadi M, Darvishi N. Substance use disorder and risk of suicidal ideation, suicide attempt and suicide death: a meta-analysis. J Public Health (Oxf) 2016;38:e282-91.

18. Akbarizadeh F, Hajivandi A, Hajivandi M, Zeidabadinejad MS. Marriage Related Suicide Fatality Rates. Iran J Psychiatry 2019;14:54-9.

19. Balint L, Osvath P, Rihmer Z, Dome P. Associations between marital and educational status and risk of completed suicide in Hungary. J Affect Disorder 2016;190:777-83.

20. Kim JW, Jung HY, Won DY, Noh JH, Shin YS, Kang TI. Suicide Trends According to Age, Gender, and Marital Status in South Korea. Omega 2019;79:90-105.

21. Nazari-Kangavari H, Shojaei A, Hashemi-Nazari SS. Suicide Mortality Trends in Four Provinces of Iran with the Highest Mortality, from 2006-2016. J Res Health Sci 2017;17:e00382.

22. Ajdacic-Gross V, Weiss MG, Ring M, Hepp U, Bopp M, Gutzwiller F, et al. Methods of suicide: international suicide patterns derived from the WHO mortality database. Bull World Health Organ 2008;86:726-32.

23. Helaly AMN, Ali EF, Zidan EM. The pattern of suicide in the Western Kingdom of Saudi Arabia: a retrospective study from 2008 to 2012. Am J Forensic Med Pathol 2015;36:27-30.

24. Göktaş S, Metintaş S. Suicide mortality trends by age, gender and method in Turkey, 2002-2015. Turk J Public Health 2019;17:195-206.

25. Abbas MJ, Alhemiary N, Razaq EA, Naosh S, Appleby L. The Iraqi national study of suicide: Report on suicide data in Iraq in 2015 and 2016. J Affect Disord 2018;229:56-62.

26. Jordans MJ, Kaufman A, Brenman NF, Adhikari RP, Luitel NP, Tol WA, et al. Suicide in South Asia: a scoping review. BMC psychiatry 2014;14:358.

27. Shojaei A, Moradi S, Alaeddini F, Khoda-Doost M, Ghadirzadeh MR, Khademi A. The association between completed suicides and season of the year in an Iranian population. Iran J Public Health 2013;42:293-7.

28. Rostami M, Jalilian A, Poorolajal J, Mahaki B. Time Series Analysis of Monthly Suicide Rates in West of Iran, 20062013. Int J Prev Med 2019;10:78. 